\title{
Molecular cloning and immune response analysis of putative variable lipoproteins from Mycoplasma mycoides subsp capri
}

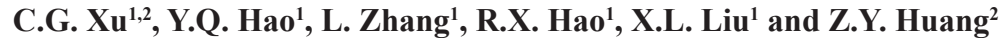

${ }^{1}$ Key Laboratory of Clinical Diagnosis and Treatment Techniques for Animal Disease, Ministry of Agriculture, Veterinary School, Inner Mongolia Agricultural University, Hohhot, Inner Mongolia, China ${ }^{2}$ Life Science \& Chemistry School, Hulunbuir College, Hulunbuir, Inner Mongolia, China

Corresponding author: Y.Q. Hao

E-mail: haoyq1960@163.com / Yongqinghao1960@yahoo.com.cn

Genet. Mol. Res. 13 (1): 1527-1539 (2014)

Received December 10, 2012

Accepted June 28, 2013

Published March 12, 2014

DOI http://dx.doi.org/10.4238/2014.March.12.5

\begin{abstract}
Mycoplasma mycoides subsp capri is the cause of goat "MAKePS" (Mastitis, Arthritis, Keratoconjunctivitis, Pneumonia, Septicemia) syndrome. We identified three genes (GL_000459; 000461; 000462) as variable lipoprotein genes in the M. mycoides subsp capri str. PG3 genome by genomic information and comparative genomic analyses. To study the role of variable lipoproteins in M. mycoides subsp capri pathogenesis and evaluate the immunogenic and protective potentials of those proteins, we constructed the expression systems and expressed the mature peptide portion of the three proteins in $E$. coli. We also determined the titers and opsonophagocytosis activity of total IgG antibodies and the levels of Th1 and Th2 cytokines in sera, and we ran a lymphocyte proliferation assay in mice immunized with recombinant proteins His-tag-GL000459, His-tag-GL000461, and His-tag-GL000462. These three lipoproteins induced humoral and cellular immune responses in the immunized mice. Additionally, the
\end{abstract}


whole blood opsonophagocitic in vitro assay demonstrated that the antibodies produced by the immunized groups can neutralize strain PG3; consequently, these three variable lipoproteins could be the major surface antigens in M. mycoides subsp capri str. PG3.

Key words: Mycoplasma mycoides subsp capri; Immune responses; Variable lipoproteins genes; Recombinant proteins; Opsonophagocytic activity

\section{INTRODUCTION}

Mycoplasma mycoides subsp capri (Mmc) mainly causes mastitis, arthritis, keratitis, pneumonia and septicaemia in goats (Thiaucourt and Bolske, 1996), which threaten a great number of goat populations throughout the world, with a high impact in Asia, Africa, and the Middle East (MacOwan and Minette, 1976; Ojo, 1976; Villalba et al., 1992; Martrenchar et al., 1995). In China, the diseases are prevalent in flocks in Guizhou province and Guangxi zhuang autonomous region, resulting in major economic losses (Wan et al., 2001; Zou et al., 2007). Mmc, together with Mycoplasma mycoides subsp mycoides Small Colony (MmmSC), belongs to the mycoides subgroup of the M. mycoides cluster (Cottew et al., 1987; Hotzel et al., 1996; Chu, 2011; Fischer et al., 2012). To date, complete genome sequences of ten strains respectively belonging to five members of the $M$. mycoides cluster have been published in GenBank. More genomic information is available. It facilitates the studies on the molecular basis and pathogenic mechanisms of infection by members of the M. mycoides cluster. Comparative genomic analysis illustrates high genomic plasticity and their very close relatedness (Thiaucourt et al., 2011; Fischer et al., 2012).

Variable lipoproteins are widespread among mycoplasma species. Vmc and $v m m$, variable lipoproteins genes of Mycoplasma capricolum subsp capricolum (Mcc) and MmmSC, respectively, encode distinct variable lipoproteins (Persson et al., 2002; Wise et al., 2006). A cluster of genes in the Mmc strain 95010 genome (MLC_9030; 9040; 9050; 9070; 9080; 9090) and Mmc strain GM12 genome (MMCAP2_0900 to 0904) sharing sequence similarities with $v m c$ may encode variable lipoproteins according to annotation (Thiaucourt et al., 2011). On the basis of comparative genomic analysis of Mycoplasma leachii (Ml) str. PG50 with other pathogens, a portfolio of phase-variable lipoprotein genes verifies the widespread presence of a stochastic mechanism of surface diversification (Wise et al., 2012). In addition, it supports the notion that variable lipoprotein genes likely play a role in host niche adaptation, immune avoidance or disease transmission (Browning et al., 2011; Chopra-Dewasthaly et al., 2012). Variable lipoproteins form a family with a highly conserved N-terminal signal peptide and cleavage site (AVIAC), some conserved stretches and a variable region in their $\mathrm{C}$-terminal part. They vary in length and display repeated AA motifs (or repeated TA motifs) in Mcc (in MmmSC, Mmc and Ml). The functions of these motifs are unknown. Maybe repeat count variation of poly AA tracts (or poly TA tracts) regulates gene expression by affecting the interaction between promoters and RNA polymerase (Le et al., 1996; Zhang and Wise, 1997; Washburn et al., 1998; Persson et al., 2002; Wise et al., 2006; Thiaucourt et al., 2011).

In this study, we sequenced the whole genome of Mmc str. PG3 and obtained more insight into Mmc. A cluster of genes (GL_000459; 000461; 000462) were annotated as vari- 
able lipoproteins genes in strain PG3 genome by genomic information analysis and comparative genomic analysis. The three putative variable lipoproteins all have a typical variable lipoprotein family-specific structure. The three genes that encode the lipoproteins were cloned into bacteria separately and the proteins were expressed in bacteria. Purified recombination proteins from bacteria were used to immunize mice. Humoral and cell-mediated immune responses to them were assessed.

\section{MATERIAL AND METHODS}

\section{Mycoplasma strain and culture}

The Mmc reference strain PG3 in this laboratory, isolated from Turkey, was obtained from China Veterinary Microbial Culture Collection (CVCC). The strain PG3 was revived and cultured in modified Hayflick's broth ( $1.0 \mathrm{~g}$ glucose, $21.0 \mathrm{~g}$ PPLO, $0.4 \mathrm{~g}$ sodium pyruvate, 10 $\mathrm{mL} \mathrm{25 \%} \mathrm{(w/v)} \mathrm{yeast} \mathrm{extract,} 200 \mathrm{~mL}$ donor equine serum (Hyclone; Logan, UT, USA), $10 \mathrm{~mL}$ $10,000 \mathrm{IU} / \mathrm{mL}$ penicillin-G, and $2.5 \mathrm{~mL} 0.4 \%(\mathrm{w} / \mathrm{v})$ phenol red, $\mathrm{pH} 7.6-7.8$ adjusted with $0.1 \mathrm{M}$ sodium hydroxide) at $37^{\circ} \mathrm{C}$, in a $5 \% \mathrm{CO}_{2}$ atmosphere for 3 days.

\section{Complete genomic DNA sequencing and genome annotation}

Genomic DNA was extracted using a mycoplasma genome DNA miniprep kit (Biomiga. Inc., USA) and sent to the Beijing Genomics Institute (Shenzhen, China) for further processing. The genome was sequenced to $100 \times$ coverage by the whole-genome shotgun (WGS) approach using paired-end Sanger sequencing. The reads were assembled using SOAP de novo. Genes were predicted using Glimmer3.0 and most were compared to the related Mmc strain 95010 genome (GenBank, accession No. Q377874). The genes were annotated through Blast searches in the databases Swiss-Prot (release: 2010-12), TrEMBL (release 2010-04), COG (release: 2009-03-31), KEGG (release: 55.1, 2010-09-01), and NCBI-NR (release: 2010-11-03). Lipoproteins were determined using preg in the EMBOSS package 6.2. In addition, signal peptide sequences were predicted using SignalP4.0.

\section{Cloning of putative variable lipoprotein genes GL_000459, GL_000461, and GL_000462 and expression of the proteins}

The isolated genomic DNA was used as a template for polymerase chain reaction (PCR) of the DNA fragments of GL_000459, GL_000461 and GL_000462. Primers including the enzyme cutting sites, designed on the basis of their mature peptide gene sequences, are listed in Table 1. PCR involved the following steps: $94^{\circ} \mathrm{C}$ for 4 min and 30 cycles of $94^{\circ} \mathrm{C}$ for $30 \mathrm{~s}$, corresponding melting temperature listed in Table 1 for $30 \mathrm{~s}$, and $72^{\circ} \mathrm{C}$ for 30 s. Finally, the reactions were incubated at $72^{\circ} \mathrm{C}$ for $10 \mathrm{~min}$. The PCR products were cloned into a pMD19-T vector (Takara Biotechnology Co., Ltd., Japan) and sequenced. The three clones harboring mature peptide sequences were identified by homological analysis using the three putative variable lipoproteins genes of Mmc strain PG3. The PCR fragments were cloned in frame into the pET-32a $(+)$ bacterial expression vector (Novagen; Madison, WI, USA). After being digested with EcoRI-XhoI and HindIII- XhoI (Takara Biotechnology Co., 
Ltd., Japan), the reconstructed vectors were designated as pET-32a (+)-MmcGL000459, pET32a (+)-MmcGL000461 and pET-32a (+)-MmcGL000462 and used for the expression of recombinant fusion proteins (His-tag-GL000459, His-tag-GL000461 and His-tag-GL000462) in Escherichia coli (E. coli) BL21 (DE3) cells (TianGen Biological Inc., China) following manufacturer instructions.

Table 1. Primers used for amplication, corresponding melting temperature of PCR program and expected amplified bands.

\begin{tabular}{llcc}
\hline Gene & Primer sequence $\left(5^{\prime} \rightarrow 3^{\prime}\right)$ & Melting temperature $\left({ }^{\circ} \mathrm{C}\right)$ & Amplified size $(\mathrm{bp})$ \\
\hline GL_000459 & Fwd: CCCGAATTCGCAGTAATTGCTTGTGGTG & & \\
& Rev: CCGCTCGAGAAGGGCTTATCTGGAACAT & 61 & 488 \\
GL_000461 & Fwd: CCGAAGCTTCAGTAATTGCTTGTGGTGATA & 61 & 817 \\
& Rev: CCGCTCGAGGGGTAGGTTGAGTTGAGTTAT & & 632 \\
GL_000462 & Fwd: GGTAAGCTTGTGCTGCAGTAATTGCTTGTGGT & & 62 \\
& Rev: CGTCTCGAGGAGACTGACGAATTATTATTTATTCTTTTGT & 62 & \\
\hline
\end{tabular}

Italic and underlined sequences indicate the restriction enzyme sites used for cloning (EcoRI-XhoI for GL_000459, HindIII-XhoI for GL_000461 and GL_000462).

\section{Purification of recombinant proteins His-tag-GL000459, His-tag-GL000461, and His-tag-GL000462}

The target fusion proteins induced by $0.5 \mathrm{mM}$ isopropyl $\beta$-D-1-thiogalactopyranoside (IPTG) for $6 \mathrm{~h}$ at $37^{\circ} \mathrm{C}$ were separated by sodium dodecyl sulfate-polyacrylamide gel electrophoresis (SDS-PAGE) on a $12 \%$ gel, followed by Coomassie R250 blue staining to evaluate protein size. They were solubilized and purified on a $5 \mathrm{~mL}$ Ni Sepharose column (HisTrap FF; GE Healthcare, Uppsala, Sweden). In addition, Western blotting was performed by using polyclonal antibodies from immunized rabbits that were generated by injection of ultrasonic whole cell proteins of strain PG3, which were confirmed by indirect enzyme-linked immunosorbent assay (ELISA). The primary antibodies in sera of immunized rabbit were detected by goat anti-rabbit IgG antibody labeled with horseradish peroxidase (HRP) (Sangon Biotech Co., Ltd.; Shanghai, China).

\section{Mouse immunization}

BALB/c mice (6-8 weeks old) were purchased from the Experimental Animal Centre, Inner Mongolia University. Experimental protocols were approved by the Research Ethics Committee of College of Veterinary Medicine, Inner Mongolia Agricultural University. The mice were then randomly divided into four groups ( 8 mice per group). Three groups were injected subcutaneously with $100 \mu \mathrm{L}$ phosphate-buffered saline (PBS) containing 50-100 $\mu \mathrm{g}$ purified recombinant proteins His-tag-GL000459, His-tag-GL000461, and His-tag-GL000462, respectively, mixed with $100 \mu \mathrm{L}$ complete Freund's adjuvant (Sigma Corporation of America). The fourth group was used as a negative control by subcutaneously injecting $100 \mu \mathrm{L}$ PBS mixed with $100 \mu \mathrm{L}$ complete Freund's adjuvant. The second and the third immunizations were intramuscularly performed on Days 10 and 21 with $100 \mu \mathrm{L}$ PBS containing 25-50 $\mu$ g purified recombinant proteins, mixed with $100 \mu \mathrm{L}$ incomplete Freund's adjuvant (Sigma Corporation of America). 


\section{Detection of injected protein-specific total IgG antibodies in mice}

Sera were collected on Days $0,14,21$, and 28 and stored at $-80^{\circ} \mathrm{C}$. The titers of serum anti-His-tag-GL000459, anti-His-tag-GL000461, and anti-His-tag-GL000462 antibodies in immunized mice were determined by indirect ELISA. Purified His-tag-GL000459, His-tagGL000461, and His-tag-GL000462 proteins, $0.5 \mu \mathrm{g}$ per well, were used to coat 96-well ELISA plates (Corning Costar, Lowell, MA, USA), which were incubated overnight at $4^{\circ} \mathrm{C}$. Plates were washed and blocked with $5 \%$ nonfat milk for $2 \mathrm{~h}$ at $37^{\circ} \mathrm{C}$. Eight serum samples from each group collected on Days 0, 14, 21, and 28 were pooled using equal volumes. The pooled serum samples were serially diluted in blocking buffer, then added to the wells and incubated for 1.5 $\mathrm{h}$ at $37^{\circ} \mathrm{C}$. The pooled serum sample of the fourth group was used as a negative control. Plates were washed before adding HRP-labeled goat anti-mouse IgG (TianGen Biological Inc.) at a 1:1000 dilution. Plates were then incubated for $1 \mathrm{~h}$ at room temperature. The plates were washed again before adding one-component 3,3',5,5'-tetramethylbenzidine substrate solution (TianGen Biological Inc.) and incubating for 5-10 $\mathrm{min}$ at $37^{\circ} \mathrm{C}$. Enzymatic reactions were stopped with $3 \mathrm{M} \mathrm{H}_{2} \mathrm{SO}_{4}$ and absorbance was recorded at $492 \mathrm{~nm}$.

\section{Detection of Th1 and Th2 cytokines in mouse sera}

Four cytokines including interleukin 2 (IL-2), IL-4, IL-10 and interferon $\gamma($ IFN- $\gamma$ ) levels were measured by ELISA with mouse IL-2, IL-4, IL-10, and IFN- $\gamma$ ELISA assay kits (Becton, Dickinson, USA), in serum samples of mice collected on Day 28. The serum samples on Day 28 of the fourth group were used as a negative control. Assays were performed following manufacturer guidelines.

\section{Lymphocyte proliferation assay}

The lymphocyte proliferative responses to His-tag-GL000459, His-tag-GL000461, and His-tag-GL000462 from a pool of splenocytes isolated from four immunized mice within each group were detected by 3-(4, 5)-dimethylthiazol(-2-yl)-3,5-diphenyltetrazolium bromide (MTT) assay. The splenocytes were cultured in RPMI-1640 in 24-well cell culture plates (Corning Costar, Lowell, MA, USA) at 4 x $10^{5}$ cells/well. Subsequently, the medium was respectively added with a final concentration of $10 \mu \mathrm{g} / \mathrm{mL}$ purified His-tag-GL000459, His-tag-GL000461, and His-tag-GL000462. Meanwhile, that from mice immunized with PBS was used as the negative control. After incubation for $60 \mathrm{~h}$ at $37^{\circ} \mathrm{C}$ in $5 \% \mathrm{CO}_{2}$, the proliferation responses were detected by the MTT dye assay. At the end of the incubation, the splenocytes in wells were centrifuged at $1000 \mathrm{rpm}$ for $5 \mathrm{~min}$ and a portion of supernatant was discarded. The splenocytes were resuspended and dimethyl sulfoxide was added. After shaking for $5 \mathrm{~min}$, the plates were read at $570 \mathrm{~nm}$. The stimulation index (SI) was calculated as the ratio of the average OD value of wells containing recombinant protein-stimulated cells to that of wells containing cells cultured with Roswell Park Memorial Institute-1640 (RPMI-1640) medium alone.

\section{Opsonophagocytosis assays}

The whole blood specimens from immunized mice were collected in sodium heparin- 
anticoagulated tubes by the heart puncture on Day 28. A volume of $200 \mu \mathrm{L}$ anticoagulated whole blood and $100 \mu \mathrm{L}$ liquid culture of strain PG3 cultured in modified Hayflick's broth for 3 days were mixed thoroughly. Anticoagulated whole blood specimens on Day 28 of the fourth group were used as a negative control. The mixture was placed on a rotary shaker (200 rpm) at $37^{\circ} \mathrm{C}$ for $3 \mathrm{~h}$. A volume of $200 \mu \mathrm{L}$ dilution (from $10^{-1}$ to $10^{-7}$ ) of the mixture was used to coat modified Hayflick's plate and incubated at $37^{\circ} \mathrm{C}$, in a $5 \% \mathrm{CO}_{2}$ atmosphere for 3 days. Colonyforming units (CFU) on plates were counted and the number of surviving strain PG3 cells mixed with whole blood specimens were calculated using the following formula (Legend 1).

\section{Statistical analysis}

Data of the opsonophagocytic assays and the levels of cytokines were analyzed using the SAS v9.1 software, and data of lymphocyte proliferation were analyzed by the unpaired one-tailed Student $t$-tests with the TTEST function in Excel. The significance threshold was set at $\mathrm{P}=0.05$ for all analyses.

\section{RESULTS}

\section{Genome sequence of Mmc str. PG3}

A 474-bp library and a 2374-bp library were constructed. We obtained $120 \mathrm{Mb}$ and $60 \mathrm{Mb}$ of clean reads ( 99.43 and $97.87 \%$ paired-end reads). The assembly produced 9 scaffolds $(>500 \mathrm{bp}$ ) and 22 non-redundant contigs in total. The numbers of total bases of the scaffolds and the contigs were $1,025,065 \mathrm{bp}$ and 1,023,884 bp, respectively. This whole genome shotgun project has been deposited at DDBJ/EMBL/GenBank under the accession ANIV00000000. The version described in this paper is the first version, ANIV01000000. The genome contained 846 putative coding sequences.

\section{Identification of variable lipoproteins from Mmc str. PG3}

The 417-, 624-, and 684-bp open reading frame (ORF) of variable lipoprotein genes GL_000459, GL_000461, and GL_000462 were identified from the Mmc str. PG3 complete genome sequence. The ORFs encoded 138-, 207-, and 227-amino acid proteins with theoretical molecular weights of $15.3,23.6$, and $25.1 \mathrm{kDa}$ and isoelectric points of 8.5, 8.8, and 9.1. The three variable lipoproteins from Mmc str. PG3 showed 91-42\% homology to those from Mmc str. 95010 and GM12. Putative translated lipoproteins of genes GL_000459, GL_000461, and GL 000462 shared approximately the same signal peptide containing 23 amino acids [MKKLLTILGSVGLM(V)ATTSAAVIA] and lipoprotein cleavage site (AVIAC) at the Nterminus, with 96-63\% sequence identity with those from the Mycoplasma mycoides cluster. Repeated TA motifs that are typical of variable lipoproteins in mycoplasmas were also found within the promoter region of the three genes. In addition, a motif TTGACAT(TA), which is also found in the upstream region of $v m m$, was located upstream of their start codon ATG.

\section{PCR amplification and cloning of genes GL_000459, GL_000461, and GL_000462}

Figure 1 shows clear amplification bands about 488, 632 and 817 bp (Lanes 1-3) for the 
three genes GL_000459, GL_000462, and GL_000461. The PCR fragments were cloned into the pMD-19T vector to generate the pMD-19T-MmcGL000459, pMD-19T-MmcGL000462, and pMD-19T-MmcGL000461 vectors. The target fragments inside amplified productions sequenced had a $100 \%$ nucleotide sequence similarity with corresponding genes by alignment, suggesting successful cloning of the putative variable lipoprotein genes GL_000459, GL_000461, and GL_000462.

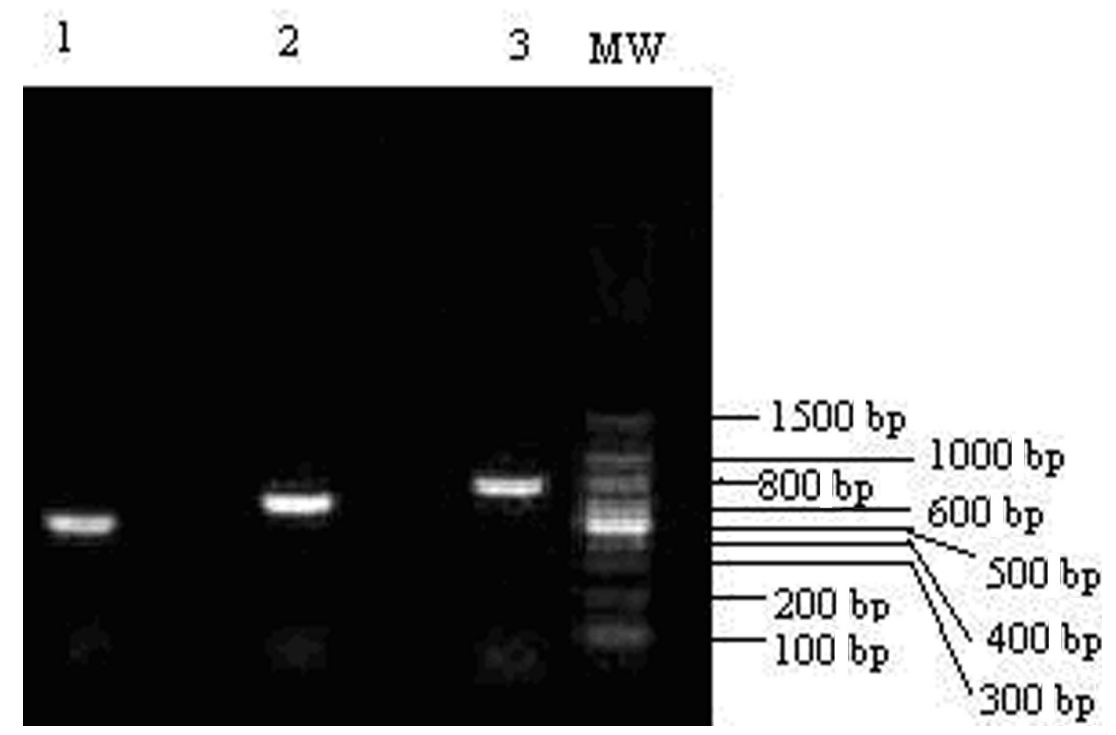

Figure 1. Ethidium bromide agarose gel images of PCR products of GL_000459, GL_000461, and GL_000462 genes using respective primers listed in Table 1 . Lane $M W=$ DNA Marker $(100 \mathrm{bp})$. Lane $1=$ PCR product of GL_000459 gene. Lane 2 = PCR product of GL_000462 gene. Lane 3 = PCR product of GL_000461 gene.

\section{Expression of the recombinant GL_000459, GL_000461, and GL_000462 proteins}

Variable lipoproteins are believed to be targets of the adaptive immune response and contribute to the distinctive antigenic profile of pathogens. Therefore, bacterial protein expression systems expressing the mature peptide portion of Mmc GL_000459, GL_000461, and GL_000462 proteins were constructed to evaluate their immunogenic and protective potential. E. coli BL21 (DE3) cells were transformed with pET-32a (+)-MmcGL000459, pET-32a (+)-MmcGL000461, and pET-32a (+)-MmcGL000462, respectively, and incubated in LB medium with ampicillin (100 $\mu \mathrm{g} / \mathrm{mL}$ ) for 18-24 $\mathrm{h}$. The level of expression and purity of the fusion proteins were analyzed by SDS-PAGE and then by Western blotting. SDS-PAGE results are shown in Figure 2A, B, C. SDSPAGE analysis of whole cell lysate demonstrated approximately 31-, 40-, and 41-kDa target bands corresponding to the expected bands. Optimum expression was obtained with incubation time of 4 $\mathrm{h}$ for them when the concentration of IPTG was $0.5 \mathrm{mM}$. The fusion proteins were solubilized. The integrity and identity of the fusion proteins could be ascertained using polyclonal antibodies in sera collected from rabbits immunized with ultrasonic whole cell proteins of strain PG3 by Western blot analysis. The results showed single bands of approximately 31, 40, and 41kDa for GL_000459, GL_000461, and GL_000462 fusion proteins, respectively (Figure 2D, E, F). 

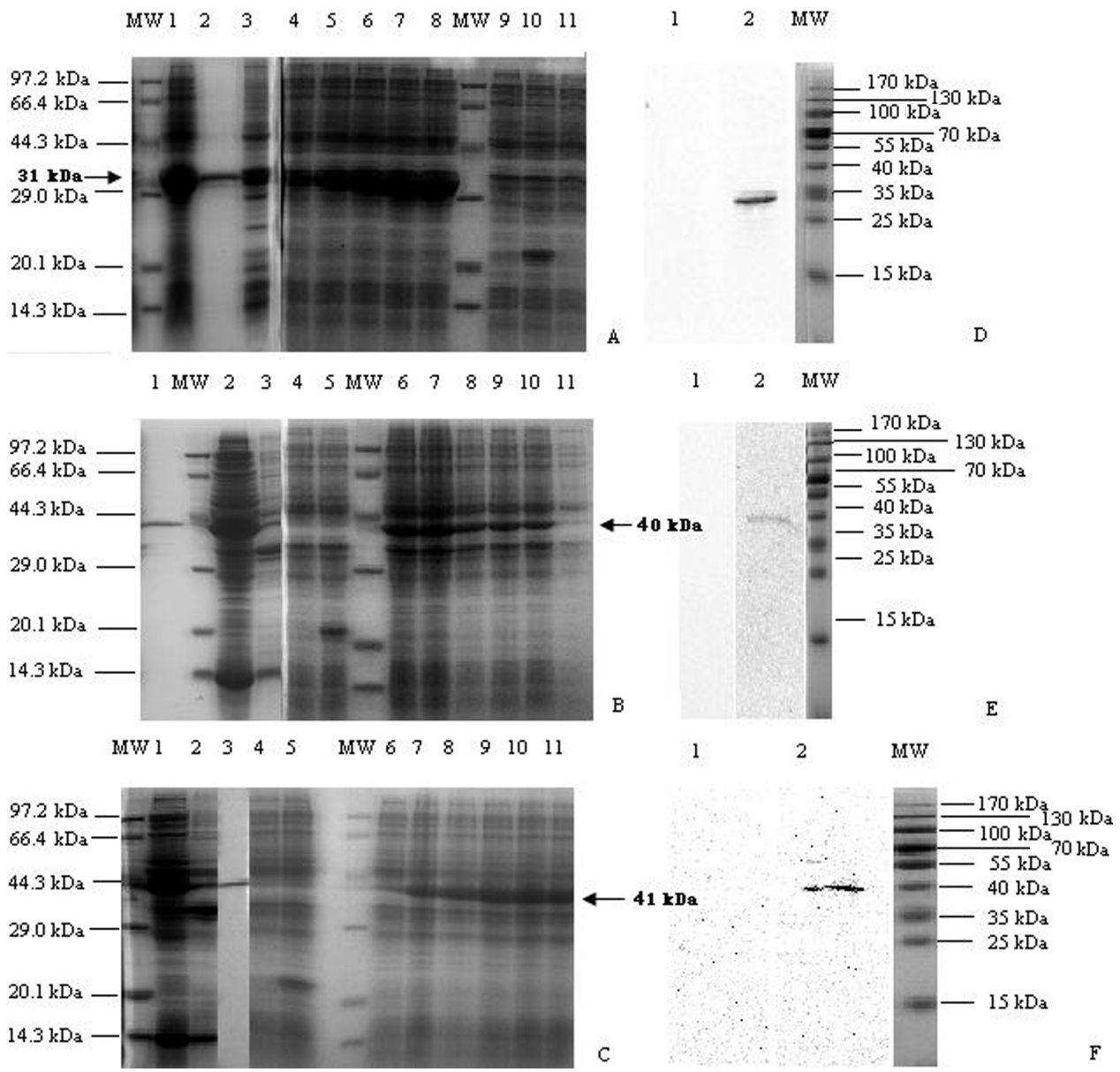

Figure 2. Expression and immunoblotting analysis of GL_000459, GL_000461, and GL_000462 fusion proteins of Mmc strain PG3. A, B, and C. The SDS-PAGE results of cell lysate derived from E. coli BL21 (DE3) transformed with pET-32a (+)-MmcGL000459, pET-32a (+)-MmcGL000461, and pET-32a (+)-MmcGL000462. D, E and F. Western blotting analysis of the expression of recombinant GL_000459, GL_000461, and GL_000462 proteins using polyclonal antibodies in sera collected from rabbits immunized with ultrasonic whole cell proteins of strain PG3. Lane $M W=$ Protein Marker (low); A. lane $1=$ pET-32a (+)-MmcGL000459/BL21 supernatant; A. lane 2, B. lane 1, C. lane 3 = Purified product after elution on a $5 \mathrm{~mL}$ Ni Sepharose column; A. lane $3=$ pET-32a (+)-MmcGL000459/ BL21 precipitate; A. lane 4-8 = expression products of 1-5 h induced with IPTG (4: $1 \mathrm{~h}, 5: 2 \mathrm{~h}, 6: 3 \mathrm{~h}, 7: 4 \mathrm{~h}, 8: 5 \mathrm{~h})$; A. lane $9=\mathrm{pET}-32 \mathrm{a}(+)-M m c G L 000459 / \mathrm{BL} 21$ induced without IPTG; A. lane 10, B. lane 5, C. lane $5=\mathrm{pET}-32 \mathrm{a}(+) /$ BL21 induced with IPTG; A. lane 11, B. lane 4, C. lane $4=\mathrm{pET}-32 \mathrm{a}(+) / \mathrm{BL} 21$ induced without IPTG; B. lane $2=$ pET-32a (+)-MmcGL000461/BL21 supernatant; B. lane 3 = pET-32a (+)-MmcGL000461/BL21 precipitate; B. lane 6-10= Expression products of 1-5 h induced with IPTG $(6: 5 \mathrm{~h}, 7: 4 \mathrm{~h}, 8: 3 \mathrm{~h}, 9: 2 \mathrm{~h}, 10: 1 \mathrm{~h})$; B. lane $11=$ pET-32a (+)-MmcGL000461 /BL21 induced without IPTG; C. lane 1 = pET-32a (+)-MmcGL000462/BL21 supernatant; C. lane $2=$ pET-32a (+)-MmcGL000462/BL21 precipitate; C. lane $6=$ pET-32a (+)-MmcGL000462/BL21 induced without IPTG; C. lane 7-11 = expression products of 1-5h induced with IPTG (7: $1 \mathrm{~h}, 8: 2 \mathrm{~h}, 9: 3 \mathrm{~h}, 10: 4 \mathrm{~h}, 11: 5 \mathrm{~h})$; D. lane 1, E. lane 1, F. lane 1 = immunoblotting analysis of recombinant GL_000459, GL_000461, and GL_000462 proteins using negative sera collected from rabbits not immunized with ultrasonic whole cell proteins of strain $\mathrm{PG} 3$ respectively; D. lane 2, E. lane 2, F. lane 2 = immunoblotting analysis of recombinant GL_000459, GL_000461, and GL_000462 proteins using positive sera collected from rabbits immunized with ultrasonic whole cell proteins of strain PG3 respectively. 


\section{Total IgG antibody titers}

His-tag-GL_000459, His-tag-GL_000461, and His-tag-GL_000462-specific IgG antibodies were only detected in pooled sera taken from vaccinated mice. Pooled sera of mice taken from the control group at the same sampling time never reacted with the three recombinant proteins. All vaccinations elicited humoral responses to the recombinant proteins His-tag-GL_000459, His-tag-GL_000461, and His-tag-GL_000462 (Figure 3). The titer of anti-His-tag-GL000461 IgG antibody was higher than that of anti-His-tag-GL000459 and anti-His-tag-GL000462 IgG antibodies after the third immunization.

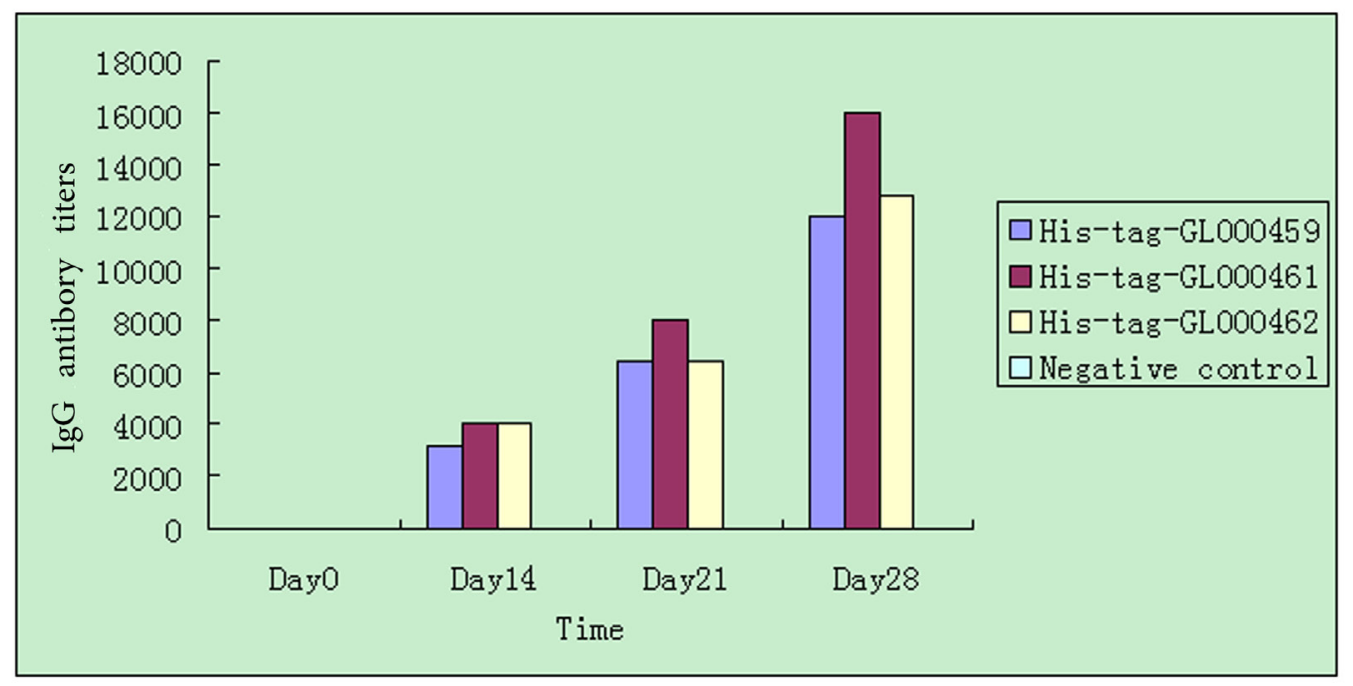

Figure 3. Total IgG antibodies response of immunized mice. BALB/c mice (6-8weeks old) were immunized with 50-100 $\mu$ g purified His-tag-GL000459, His-tag-GL000461, and His-tag-GL000462 on Day 0. The second and the third immunizations were performed on Day 10 and Day 21 with 25-50 $\mu \mathrm{g}$ purified recombinant proteins. Mice immunized with PBS were used as a negative control. Mouse sera were sampled on Day 0, Day 14, Day 21, and Days 28 and the IgG titers were tested by indirect ELISA.

\section{Levels of Th1 and Th2 cytokines}

After last immunization, the immune response was investigated using double-antibody sandwich ELISA that measured simultaneously the amount of four cytokines including IL-2, IL-4, IL-10, and IFN- $\gamma$ in the sera of the immunized mice. It is known that IL-2 and IFN- $\gamma$ belong to the Th1 cytokines. Th1 cytokines play a role in the cellular immune response. And IL-4 and IL-10, which belong to Th2 cytokines, act as regulators in humoral and adaptive immunity. As shown in Figure 4, the levels of four cytokines in the sera of the mice immunized with His-tag-GL000459, His-tag-GL000461, and His-tag-GL000462 were all higher than that of the control group. Immunization with His-tag-GL000459 significantly increased the production of IL-2 and IL-4 $(\mathrm{P}<0.05)$, and slightly increased that of IL-10 and IFN- $\gamma(\mathrm{P}>$ 0.05). Immunization with His-tag-GL000461 significantly increased that of all these cytokines $(\mathrm{P}<0.05)$. Immunization with His-tag-GL000462 significantly increased that of IL-2, IL-4, 
and IFN- $\gamma(\mathrm{P}<0.05)$, and slightly increased that of IL- $10(\mathrm{P}>0.05)$. Among the three treated groups, the His-tag-GL000461-immunized group showed the highest levels of cytokines. Therefore, these findings may indicate that the three recombinant proteins exert both cellular and humoral immunity response to them in mice by increasing the levels of several cytokines, including IL-2, IL-4, IL-10, and IFN- $\gamma$.

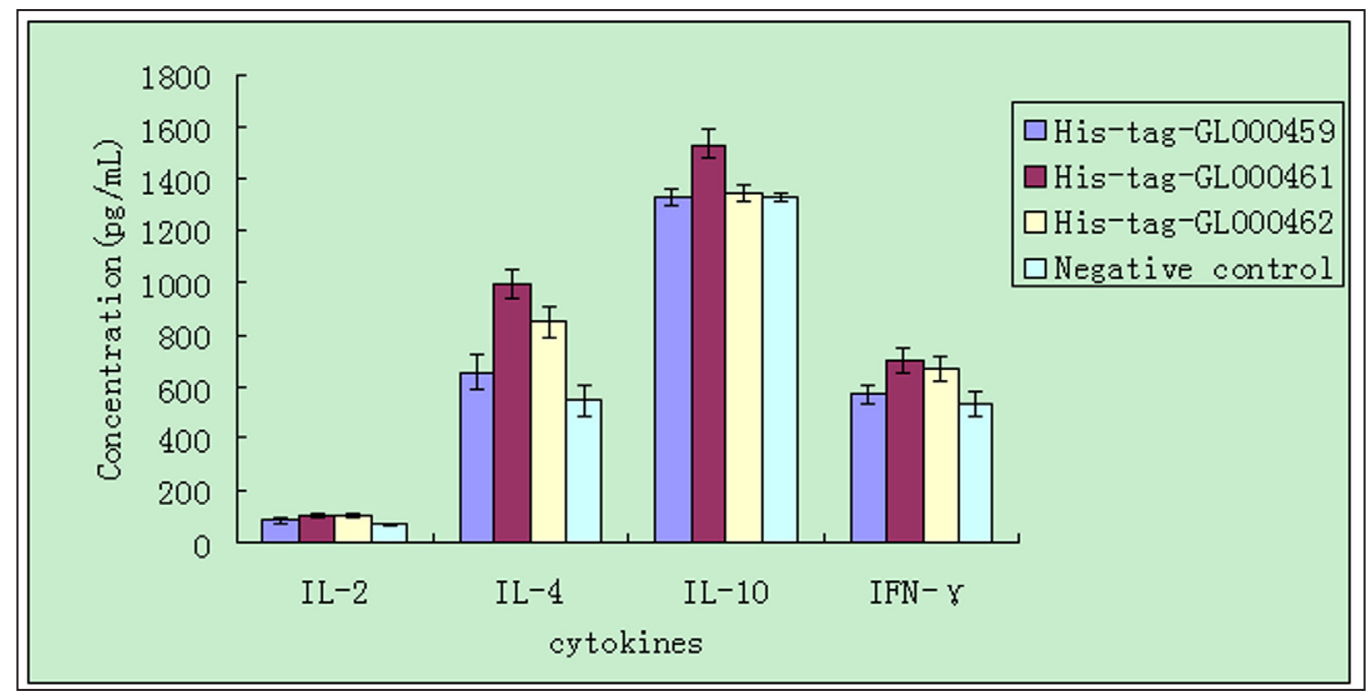

Figure 4. Analysis of serum IL-2, IL-4, IL-10, and INF- $\gamma$ from mice immunized with His-tag-GL000459, His-tagGL000461, His-tag-GL000462 and PBS. The levels of IL-2, IL-4, IL-10, and INF- $\gamma$ in samples of mouse serum on Day 28 were measured by double-antibody sandwich ELISA. Mice immunized with PBS were used as a negative control. Cytokines of 8 mice sera were titrated for each group.

\section{Lymphocyte proliferation}

To determine whether His-tag-GL000459, His-tag-GL000461, and His-tagGL000462-specific proliferation responses were induced in the immunized mice, splenic lymphocyte proliferation was measured. The stimulation index (SI) of splenic lymphocyte proliferation stimulated with His-tag-GL000459, His-tag-GL000461, and His-tag-GL000462 in the groups of immunized mice with the corresponding one were respectively $1.43 \pm 0.01$, $1.87 \pm 0.15$, and $1.61 \pm 0.13$ (Table 2). The three treated groups immunized with recombinant proteins showed significantly higher proliferation responses than their control groups immunized with PBS $(\mathrm{P}<0.05)$. Among them, His-tag-GL000461 had the strongest influence on splenic lymphocyte proliferation in mice $(\mathrm{P}<0.05)$. Therefore, His-tag-GL000459, His-tagGL000461, and His-tag-GL000462-specific splenic lymphocyte proliferation was efficiently induced by vaccination with the three recombinant proteins.

\section{Power of the whole blood opsonophagocytosis}

To study the activity of functional antibodies induced by His-tag-GL000459, His- 
tag-GL000461, and His-tag-GL000462 in mice, in vitro opsonophagocytic assays in whole blood were performed. As shown in Figure 5, the numbers of surviving strain PG3 cells mixed with whole blood with anti-His-tag-GL000459 antibodies, anti-His-tag-GL000461 antibodies, and anti-His-tag-GL000462 antibodies were all significantly less than that mixed with whole blood from mice immunized with PBS (P < 0.05). Immunization with His-tag-GL000459, His-tag-GL000461, and His-tag-GL000462 all improved opsonophagocytic activity of whole blood. The His-tag-GL000459-immunized group showed the strongest capacity of opsonophagocytosis against strain PG3, but showed no significant difference with the others $(\mathrm{P}>$ 0.05). It is suggested that anti-His-tag-GL000459, anti-His-tag-GL000461, and anti-His-tagGL000462 antibodies can clear Mmc strain PG3 infection effectively by opsonophagocytosis.

Table 2. Effect of recombinant proteins His-tag-GL000459, His-tag-GL000461, His-tag-GL000462 on spleniclymphocyte proliferation in mice (Stimulation index, SI).

\begin{tabular}{lccc}
\hline & SI $_{\text {His-tag-GL000459 }}$ & SI $_{\text {His-tag-GL000461 }}$ & SI $_{\text {His-tag-GL000462 }}$ \\
\hline Treated group immunized with recombinant proteins & $1.43 \pm 0.01$ & $1.87 \pm 0.15$ & $1.61 \pm 0.13$ \\
Control group immunized with PBS & $1.00 \pm 0.10$ & $0.96 \pm 0.09$ & $1.04 \pm 0.09$ \\
\hline
\end{tabular}

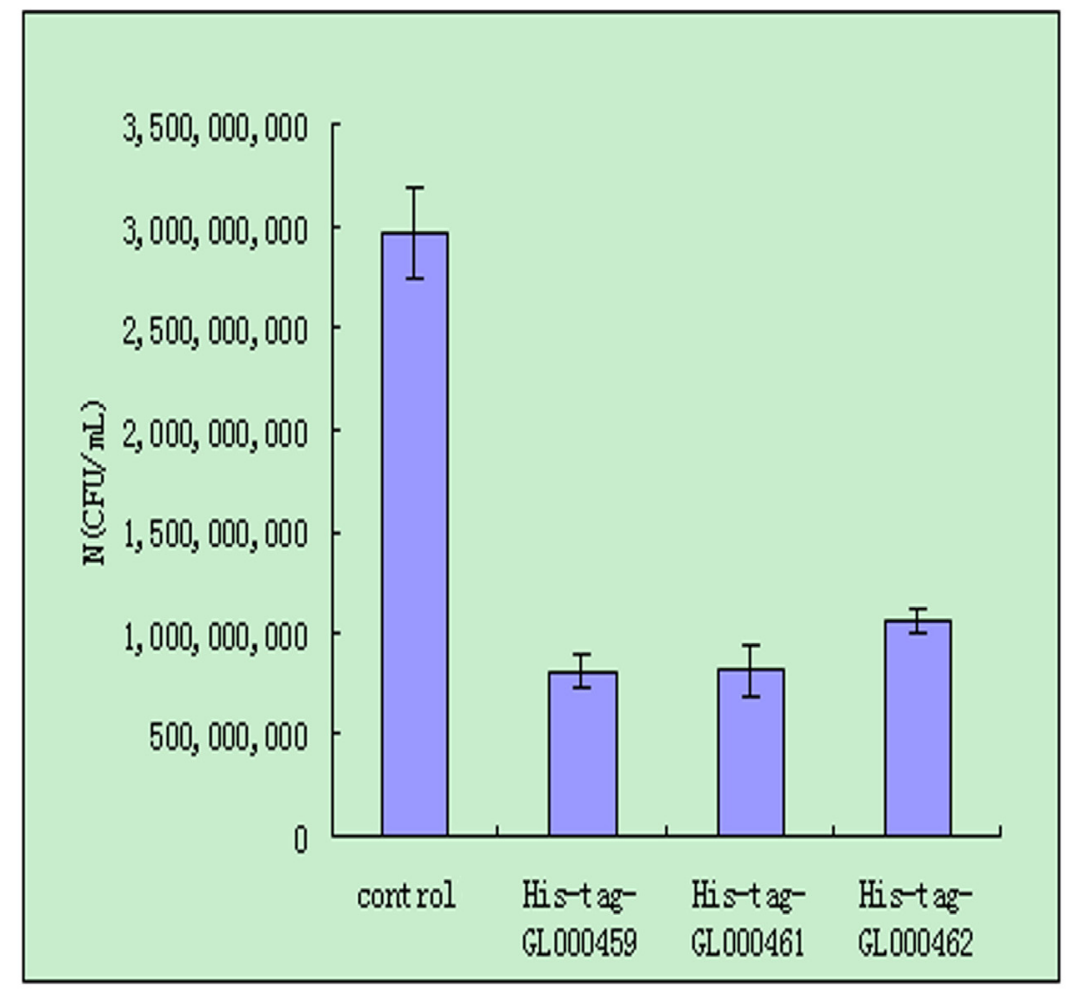

Figure 5. Opsonophagocytosis activity of whole blood from immunized mice. The whole blood specimens were collected from mice immunized with His-tag-GL000459, His-tag-GL000461, His-tag-GL000462, and PBS by the method of heart puncture on Day 28. The whole blood specimens from mice immunized with PBS were used as a negative control. The number of survival strain PG3 cell mixed with whole blood specimens (N) was calculated. 


\section{DISCUSSION}

The chronic pneumonia/pleuropneumonia caused by Mmc is one of the major health problems in goats and results in emaciation delayed market weight and infertility. The pathogenic mechanisms involved in the development of lung lesions after infection with Mmc are largely unknown. However, it is generally believed that variable lipoproteins could be involved in pathogen colonization and adaption to the host tissue environment at various stages of infection. Mmc possesses a large family of variable lipopropteins. Up to now, members of the lipoprotein gene family include MLC_9030 - 9050 and MLC_9070 - 9090 in the Mmc strain 95010 genome, MMCAP2_0900 0904 in the Mmc strain GM12 genome, and LPPA genes (Dedieu et al., 2010) in the Mmc genome. In this study, GL_000459, GL_000461, and GL_000462 were regarded as variable lipoproteins genes in the strain PG3 genome regarding a typical variable lipoproteins family-specific structure, namely, a highly conserved $\mathrm{N}$-terminal signal peptide and lipoprotein cleavage site (AVIAC), some conserved stretches and a variable region in their C-terminus and repeated TA motifs within the promoter region of the three genes. In addition, they share high amino acid sequence similarity with members of the lipoprotein gene family in strains 95010 and GM12.

The putative variable lipoproteins genes GL_000459, GL_000461, and GL_000462 were cloned and successfully expressed in E. coli systems. Three solubilized recombinant proteins of high purity were obtained. The expressed recombinant proteins could be recognized by antibodies of immunized rabbits, which indicated that they harbored same antigenic epitopes as strain PG3.

The titers and the opsonophagocytosis analysis of total IgG antibodies were tested to assess humoral responses to recombinant proteins His-tag-GL000459, His-tag-GL000461, and His-tagGL000462 in mice. In addition, cellular responses were evaluated by detecting the levels of Th1 and Th2 cytokines in sera and the ability of the antigen-specific memory lymphocytes to proliferate. The production of His-tag-GL000459, His-tag-GL000461, and His-tag-GL000462-specific antibodies, the increase in the levels of Th1 and Th2 cytokines in serum and the lymphocyte proliferation in spleen in mice immunized with His-tag-GL000459, His-tag-GL000461, and His-tagGL000462 further confirmed that His-tag-GL000459, His-tag-GL000461, and His-tag-GL000462 were expressed and induced significant immune responses to them in the immunized mice.

Since opsoni-mediated killing is important to host defense against mycoplasma infection, the opsonophagocytic activity of the anti-His-tag-GL000459 antibodies, anti-Histag-GL000461 antibodies, and anti-His-tag-GL000462 antibodies against Mmc strain PG3 is assumed to be a reliable correlate of protection. Data of in vitro opsonophagocytic assays in whole blood indicate that the immunized groups produced opsonophagocytic killing antibodies. This suggested that the three variable lipoproteins probably are relevant surface antigens in Mmc str. PG3 and are immunogenic in a natural infection.

It must also be noted that the data in this study cannot represent the picture of the complex immune responses to His-tag-GL000459, His-tag-GL000461, and His-tag-GL000462 in goats. Therefore, we cannot obviously make general predictions based on the immune responses of mice, and further research on their potential function during infection caused by Mmc in goats will be performed in the future.

\section{Conflicts of interest}

The authors declare no conflict of interest. 


\section{ACKNOWLEDGMENTS}

Research supported by the National Key Technology R\&D Program of China (\#2011BAD18B01), and the Research Award Fund for the Scientific and Technological Innovation Leading Project of Inner Mongolia Autonomous Region (\#20101808).

\section{REFERENCES}

Browning GF, Marenda MS, Noormohammadi AH and Markham PF (2011). The central role of lipoproteins in the pathogenesis of mycoplasmoses. Vet. Microbiol. 153: 44-50.

Chopra-Dewasthaly R, Baumgartner M, Gamper E, Innerebner C, et al. (2012). Role of Vpma phase variation in Mycoplasma agalactiae pathogenesis. FEMS Immunol. Med. Microbiol. 66: 307-322.

Chu YF (2011). An Etiological and Epidemiological Study on Contagious Caprine Pleuropneumoniae in China and Development of a Bacterin Against it. Doctoral thesis. China Academy of Agricultural Sciences, Beijing.

Cottew GS, Breard A, DaMassa AJ, Erno H, et al. (1987). Taxonomy of the Mycoplasma mycoides cluster. Isr. J. Med. Sci. 23: 632-635.

Dedieu L, Totte P, Rodrigues V, Vilei EM, et al. (2010). Comparative analysis of four lipoproteins from Mycoplasma mycoides subsp. mycoides Small Colony identifies LppA as a major T-cell antigen. Comp Immunol. Microbiol. Infect. Dis. 33: 279-290.

Fischer A, Shapiro B, Muriuki C, Heller M, et al. (2012). The origin of the 'Mycoplasma mycoides cluster' coincides with domestication of ruminants. PLoS One 7: e36150.

Hotzel H, Sachse K and Pfutzner H (1996). A PCR scheme for differentiation of organisms belonging to the Mycoplasma mycoides cluster. Vet. Microbiol. 49: 31-43.

Le GD, Solsona M, Rosengarten R and Poumarat F (1996). Adaptive surface antigen variation in Mycoplasma bovis to the host immune response. FEMS Microbiol. Lett. 144: 267-275.

MacOwan KJ and Minette JE (1976). A mycoplasma from acute contagious caprine pleuropneumonia in Kenya. Trop. Anim. Health Prod. 8: 91-95.

Martrenchar A, Bouchel D and Zoyem N (1995). Isolation and experimental studies of Mycoplasma mycoides subsp. mycoides LC and Mycoplasma ovipneumoniae in goats in northern Cameroon. Small Rumin. Res. 16: 179-184.

Ojo MO (1976). Caprine pneumonia in Nigeria. I. Epidemiology and bacterial flora of normal and diseased respiratory tracts. Trop. Anim. Health Prod. 8: 85-89.

Persson A, Jacobsson K, Frykberg L, Johansson KE, et al. (2002). Variable surface protein Vmm of Mycoplasma mycoides subsp. mycoides small colony type. J. Bacteriol. 184: 3712-3722.

Thiaucourt F and Bolske G (1996). Contagious caprine pleuropneumonia and other pulmonary mycoplasmoses of sheep and goats. Rev. Sci. Tech. 15: 1397-1414.

Thiaucourt F, Manso-Silvan L, Salah W, Barbe V, et al. (2011). Mycoplasma mycoides, from "mycoides Small Colony" to "capri". A microevolutionary perspective. BMC Genomics 12: 114.

Villalba EJ, Poveda JB, Fernandez A, Rodriguez JL, et al. (1992). An outbreak caused by Mycoplasma mycoides species in goats in the Canary Islands. Vet. Rec. 130: 330-331.

Wan YY, Long A, Wan QJ, Pan SH, et al. (2001). Isolation and identification of the pathogens of contagious caprine pleuropneumonia in Guizhou province of China. Chin. Herb. 3: 44-46.

Washburn LR, Weaver KE, Weaver EJ, Donelan W, et al. (1998). Molecular characterization of Mycoplasma arthritidis variable surface protein MAA2. Infect. Immun. 66: 2576-2586.

Wise KS, Foecking MF, Roske K, Lee YJ, et al. (2006). Distinctive repertoire of contingency genes conferring mutationbased phase variation and combinatorial expression of surface lipoproteins in Mycoplasma capricolum subsp. capricolum of the Mycoplasma mycoides phylogenetic cluster. J. Bacteriol. 188: 4926-4941.

Wise KS, Calcutt MJ, Foecking MF, Madupu R, et al. (2012). Complete genome sequences of Mycoplasma leachii strain PG50 ${ }^{\mathrm{T}}$ and the pathogenic Mycoplasma mycoides subsp. mycoides small colony biotype strain Gladysdale. $J$. Bacteriol. 194: 4448-4449.

Zhang Q and Wise KS (1997). Localized reversible frameshift mutation in an adhesin gene confers a phase-variable adherence phenotype in mycoplasma. Mol. Microbiol. 25: 859-869.

Zou LB, Guo JG, Liu Q and Lu LQ (2007). Isolation and identification of the pathogens of contagious caprine pleuropneumonia in Guangxi of China. Shanghai J. Anim. Husb. Vet. Med. 4: 16-19. 